\title{
CONTACT STRUCTURES ON PRINCIPAL CIRCLE BUNDLES
}

\author{
FAN DING AND HANSJÖRG GEIGES
}

\begin{abstract}
We describe a necessary and sufficient condition for a principal circle bundle over an even-dimensional manifold to carry an invariant contact structure. As a corollary it is shown that all circle bundles over a given base manifold carry an invariant contact structure, only provided the trivial bundle does. In particular, all circle bundles over 4-manifolds admit invariant contact structures. We also discuss the Bourgeois construction of contact structures on odd-dimensional tori in this context, and we relate our results to recent work of Massot, Niederkrüger and Wendl on weak symplectic fillings in higher dimensions.
\end{abstract}

\section{INTRODUCTION}

The study of invariant contact structures on principal $S^{1}$-bundles can be traced back to the work of Boothby and Wang [2], cf. [7, Section 7.2]. They observed that if the Euler class of the bundle can be represented by a symplectic form on the base, a suitable connection 1-form will be an invariant contact form. Their main result was that any contact form whose Reeb vector field is regular essentially arises in this way. These contact structures are transverse to the $S^{1}$-fibres.

The classification of invariant contact structures on $S^{1}$-bundles over surfaces was obtained by Lutz [13. In the present paper we extend his results to higher dimensions. We derive a necessary (Proposition 3.6) and sufficient (Theorem 4.1) condition for an $S^{1}$-bundle to admit an invariant contact structure. Our explicit way of building an invariant contact structure from a certain symplectic splitting of the base manifold is inspired by the work of Giroux [9] on convex hypersurfaces (corresponding to $\mathbb{R}$-invariant contact structures), and it extends a construction by Stipsicz and the second author [8] from trivial to nontrivial bundles. In fact, as a consequence of our existence criterion we can show that if the trivial $S^{1}$-bundle over a given base manifold admits an invariant contact structure, then the same is true for all nontrivial $S^{1}$-bundles (Corollary 5.1). Combining this with the main result from [8] we conclude that all $S^{1}$-bundles over 4 -manifolds carry invariant contact structures.

In Section 5.3 we discuss the relation of the results in the present paper with Bourgeois's construction of $T^{2}$-invariant contact structures on $N \times T^{2}$, where $N$ is any closed contact manifold, starting from an open book decomposition of $N$.

In Section 5.2 we demonstrate by an example that the topology of the total space of the $S^{1}$-bundle is not, in general, determined by the symplectic splitting of the base. However, as we prove in Section 6, once the topology of the bundle is fixed, the symplectic splitting of the base determines the invariant contact structure on the

2010 Mathematics Subject Classification. 53D35, 55R25, 57R17, 57R22.

F. D. partially supported by grant no. 10631060 of the National Natural Science Foundation of China and a DAAD - K. C. Wong fellowship, grant no. A/09/99005, at the Universität zu Köln. 
$S^{1}$-bundle up to equivariant diffeomorphism. This classification result is formulated in terms of ideal Liouville domains, a notion introduced by Giroux and discussed at length by Massot et al. 14.

\section{Conventions}

This section merely serves to fix our normalisation conventions regarding principal connections. Let $\pi: M \rightarrow B$ be a principal $S^{1}$-bundle. Write $\partial_{\theta}$ for the vector field on $M$ generating the $S^{1}$-action. By a connection 1-form $\psi$ we mean an $S^{1}$-invariant form on $M$, i.e. $L_{\partial_{\theta}} \psi \equiv 0$, normalised by $\psi\left(\partial_{\theta}\right) \equiv 1$. Up to a factor $2 \pi$ this $\psi$ is what Bott-Tu [3] call the global angular form.

The 2 -form d $\psi$ is then $S^{1}$-invariant and horizontal, where the latter means that $i_{\partial_{\theta}} \mathrm{d} \psi \equiv 0$. It follows that $\mathrm{d} \psi$ induces a closed 2 -form $\omega$ on $B$, that is, $\mathrm{d} \psi=$ $\pi^{*} \omega$. This 2-form $\omega$ is called the curvature form of the connection $\psi$. The (real) Euler class of the $S^{1}$-bundle is the cohomology class given by $e=-[\omega / 2 \pi] \epsilon$ $H_{\mathrm{dR}}^{2}(B)$, where $H_{\mathrm{dR}}^{*}$ denotes de Rham cohomology. This Euler class is an integral cohomology class in the sense that it lives in the image of the inclusion $H^{2}(B ; \mathbb{Z}) \subset$ $H_{\mathrm{dR}}^{2}(B)$.

Given a further closed 2-form $\omega^{\prime}$ on $B$ with $\left[\omega^{\prime}\right]=[\omega] \in H_{\mathrm{dR}}^{2}(B)$, one can find a connection 1-form $\psi^{\prime}$ with $\mathrm{d} \psi^{\prime}=\pi^{*} \omega^{\prime}$. Indeed, we have $\omega^{\prime}=\omega+\mathrm{d} \gamma$ for some 1 -form $\gamma$ on $B$, and we may then set $\psi^{\prime}=\psi+\pi^{*} \gamma$. The difference between two connection 1-forms with the same curvature form is a closed horizontal 1-form.

By slight abuse of notation we shall not usually distinguish between an $S^{1}$ invariant and horizontal differential form on $M$ and the induced form on $B$.

Principal $S^{1}$-bundles over $B$ are classified by their Euler class in integral cohomology $H^{2}(B ; \mathbb{Z})$. The real Euler class $e \in H_{\mathrm{dR}}^{2}(B)$ does not completely classify $S^{1}$-bundles in the presence of torsion in $H^{2}(B ; \mathbb{Z})$; there are nontrivial $S^{1}$-bundles with flat connections, see [12. This issue will be of relevance in Section 6 only.

\section{SympleCtic DeCOMPositions}

Assume now that $B$ is a closed, connected, oriented $2 n$-manifold and $\pi: M \rightarrow B$ a principal $S^{1}$-bundle as before, with the corresponding orientation on $M$. Suppose that $M$ admits an $S^{1}$-invariant cooriented contact structure $\xi=\operatorname{ker} \alpha$ such that $\alpha \wedge(\mathrm{d} \alpha)^{n}$ is a (positive) volume form for this orientation of $M$. The contact form $\alpha$ may likewise be taken to be $S^{1}$-invariant, for we can always pass to the averaged contact form

$$
\int_{\theta \in S^{1}} \theta^{*} \alpha
$$

Then $u:=\alpha\left(\partial_{\theta}\right)$ defines a smooth $S^{1}$-invariant function on $M$. With $\psi$ a connection 1-form on $M$, define a 1-form $\beta$ on $M$ by

$$
\alpha=\beta+u \psi \text {. }
$$

This $\beta$ is $S^{1}$-invariant and horizontal. Thus, both the function $u$ and the 1 -form $\beta$ descend to $B$.

Write $\omega$ for the curvature form of $\psi$ as in the previous section.

Lemma 3.1. The $2 n$-form

$$
\Omega:=(\mathrm{d} \beta+u \omega)^{n-1} \wedge[n \beta \wedge \mathrm{d} u+u(\mathrm{~d} \beta+u \omega)]
$$

is a volume form on $B$. 
Proof. A straightforward computation gives $\alpha \wedge(\mathrm{d} \alpha)^{n}=\psi \wedge \Omega$.

The terminology in the following definition is chosen because of the obvious analogy with the theory of $\mathbb{R}$-invariant contact structures near convex hypersurfaces in the sense of Giroux [9, cf. [10, Definition 4.3].

Definition 3.2. The dividing set of $B$ induced by the contact structure $\xi$ is the set

$$
\Gamma:=\{p \in B: u(p)=0\}
$$

We write

$$
B_{ \pm}:=\{p \in B: \pm u(p) \geq 0\},
$$

so that $B \backslash \Gamma=\operatorname{Int}\left(B_{+}\right) \sqcup \operatorname{Int}\left(B_{-}\right)$.

In [13] it was shown that for $\operatorname{dim} B=2$ this dividing set classifies invariant contact structures up to equivariant diffeomorphism. The importance of this dividing set even for the classification of non-invariant contact structures on circle bundles over surfaces was observed by Giroux [10].

Lemma 3.3. The dividing set $\Gamma$ is a (possibly empty) closed codimension 1 submanifold of $B$. The 1-form $\beta_{0}:=\left.\beta\right|_{T \Gamma}$ is a contact form on $\Gamma$, inducing the orientation of $\Gamma$ as the boundary of $B_{+}$.

Proof. From the preceding lemma it follows that

$$
-\mathrm{d} u \wedge \beta \wedge(\mathrm{d} \beta)^{n-1}>0
$$

along $\Gamma$, and $-\mathrm{d} u$ evaluates positively on vectors pointing out of $B_{+}$.

We now want to show that there are symplectic forms on $B_{ \pm}$representing the Euler class of the bundle and compatible with the contact structure ker $\beta_{0}$ on the boundary in the sense of the following definition.

Definition 3.4. Let $(W, \omega)$ be a compact symplectic manifold of dimension $2 n$, oriented by the symplectic form $\omega$, and $\eta=\operatorname{ker} \beta_{0}$ a cooriented (and hence oriented) contact structure on $\partial W$ inducing the boundary orientation. We say that $(W, \omega)$ is a weak filling of $(\partial W, \eta)$ if

$$
\left.\left(\mathrm{d} \beta_{0}\right)^{k} \wedge \omega^{n-1-k}\right|_{\eta}>0 \text { for all } k=0, \ldots, n-1 .
$$

Observe that this condition does not depend on the choice of contact form for $\eta$ (among forms inducing the given coorientation). This definition of a weak filling is essentially the one proposed by Massot et al. 14 (in contrast with earlier definitions, where only $\left.\omega^{n-1}\right|_{\eta}>0$ had been required). In fact, they demand

$\left.\left(\mathrm{w}_{2}\right) \quad\left(b \mathrm{~d} \beta_{0}+\omega\right)^{n-1}\right|_{\eta}>0$ for all smooth functions $b: \partial W \rightarrow \mathbb{R}_{0}^{+}$,

which on the face of it is weaker than $\left(\mathrm{w}_{1}\right)$. Lemma 4.2 shows that by a modification in a collar one can pass from $\left(\mathrm{w}_{2}\right)$ to $\left(\mathrm{w}_{1}\right)$, see Remark 4.3 This observation can be phrased as follows.

Proposition 3.5. Weak symplectic fillability in the sense of $\left(\mathrm{w}_{2}\right)$ is equivalent to fillability in the sense of $\left(\mathrm{w}_{1}\right)$.

So for our purposes $\left(\mathrm{w}_{1}\right)$ and $\left(\mathrm{w}_{2}\right)$ can be used interchangeably. Beware, though, that a given symplectic filling may satisfy $\left(\mathrm{w}_{2}\right)$, but not $\left(\mathrm{w}_{1}\right)$. 
Proposition 3.6. Given a principal $S^{1}$-bundle $\pi: M \rightarrow B$ of Euler class e with an $S^{1}$-invariant contact structure $\xi$, then with notation as above the following holds. There are symplectic forms $\omega_{ \pm}$on $\pm B_{ \pm}$, where $-B_{-}$denotes $B_{-}$with reversed orientation, such that

(i) $\mp\left[\omega_{ \pm} / 2 \pi\right]=\left.e\right|_{B_{ \pm}}$;

(ii) if $\Gamma$ is non-empty, then $\left( \pm B_{ \pm}, \omega_{ \pm}\right)$are weak fillings of $\left(\Gamma, \operatorname{ker} \beta_{0}\right)$.

Remark 3.7. As we shall see presently, an $S^{1}$-invariant contact form $\alpha$ on $M$ gives rise in a natural way to symplectic forms $\omega_{ \pm}$on the interior of $\pm B_{ \pm}$. The symplectic forms in the statement of the proposition are obtained by considering a diffeomorphic copy of $\pm B_{ \pm}$obtained by shrinking a collar neighbourhood of its boundary. Conversely, in the proof of Theorem 4.1. where we construct an $S^{1}$ invariant contact form on $M$ from symplectic forms on $\pm B_{ \pm}$, we actually insert a 'neck' $[-1,1] \times \Gamma$ between $B_{+}$and $B_{-}$and find a contact structure on the corresponding $S^{1}$-bundle over this enlarged base manifold. The approach in Section 6 via so-called ideal Liouville domains circumvents these subtleties.

Proof of Proposition 3.6. On $B \backslash \Gamma$ one can write the volume form $\Omega$ from Lemma 3.1 as

$$
\Omega=u^{n+1}(\mathrm{~d}(\beta / u)+\omega)^{n}
$$

so

$$
\omega_{ \pm}:= \pm\left.(\mathrm{d}(\beta / u)+\omega)\right|_{\operatorname{Int}\left(B_{ \pm}\right)}
$$

are symplectic forms on $\operatorname{Int}\left(B_{ \pm}\right)$, respectively, inducing the positive orientation on $\operatorname{Int}\left(B_{+}\right)$and the negative orientation on $\operatorname{Int}\left(B_{-}\right)$. Moreover, we have

$$
\mp\left[\omega_{ \pm} / 2 \pi\right]=-\left.[\omega / 2 \pi]\right|_{\operatorname{Int}\left(B_{ \pm}\right)}=\left.e\right|_{\operatorname{Int}\left(B_{ \pm}\right)} .
$$

If $\Gamma \neq \emptyset$, we may choose $\varepsilon>0$ so small that

$$
B_{ \pm}^{\varepsilon}:=\{p \in B: \pm u(p) \geq \varepsilon\}
$$

is an isotopic copy of $B_{ \pm}$in $B$, and such that for each $s \in[-\varepsilon, \varepsilon]$ the set

$$
\Gamma_{s}:=\{p \in B: u(p)=s\}
$$

is an isotopic copy of $\Gamma$ in $B$ with $\beta_{s}:=\left.\beta\right|_{T \Gamma_{s}}$ a contact form. In other words, $B_{ \pm}^{\varepsilon}$ is obtained from $B_{ \pm}$by shrinking a collar neighbourhood of its boundary. By Gray stability [7. Theorem 2.2.2], the contact manifolds $\left(\Gamma, \operatorname{ker} \beta_{0}\right)$ and $\left(\Gamma_{ \pm \varepsilon}, \operatorname{ker} \beta_{ \pm \varepsilon}\right)$ are diffeomorphic.

On $\Gamma_{\varepsilon}$ we have for all $k=0, \ldots, n-1$, possibly after choosing a smaller $\varepsilon>0$,

$$
\left.\beta_{\varepsilon} \wedge\left(\mathrm{d} \beta_{\varepsilon}\right)^{k} \wedge \omega_{+}^{n-1-k}\right|_{T \Gamma_{\varepsilon}}=\left.\beta \wedge(\mathrm{d} \beta)^{k} \wedge(\mathrm{d} \beta / \varepsilon+\omega)^{n-1-k}\right|_{T \Gamma_{\varepsilon}}>0,
$$

so $\left(B_{+}^{\varepsilon},\left.\omega_{+}\right|_{B_{+}^{\varepsilon}}\right)$ is a weak filling of $\left(\Gamma_{\varepsilon}, \operatorname{ker} \beta_{\varepsilon}\right) \cong\left(\Gamma, \operatorname{ker} \beta_{0}\right)$. Condition (i) holds under the obvious diffeomorphism between $B_{+}^{\varepsilon}$ and $B_{+}$.

Similarly, on $\Gamma_{-\varepsilon}$ we have

$$
\left.\beta_{-\varepsilon} \wedge\left(\mathrm{d} \beta_{-\varepsilon}\right)^{k} \wedge \omega_{-}^{n-1-k}\right|_{T \Gamma_{-\varepsilon}}=\left.\beta \wedge(\mathrm{d} \beta)^{k} \wedge(\mathrm{d} \beta / \varepsilon-\omega)^{n-1-k}\right|_{T \Gamma_{-\varepsilon}}>0,
$$

so $\left(-B_{-}^{\varepsilon},\left.\omega_{-}\right|_{B_{-}^{\varepsilon}}\right)$ is a weak filling of $\left(\Gamma_{-\varepsilon}, \operatorname{ker} \beta_{-\varepsilon}\right) \cong\left(\Gamma, \operatorname{ker} \beta_{0}\right)$ in the sense of $\left(\mathrm{w}_{1}\right)$. 


\section{Constructing an invariant COntact Structure}

We are now going to show that the conditions listed in Proposition 3.6 are in fact also sufficient for the existence of an $S^{1}$-invariant contact structure on $M$.

Theorem 4.1. Let $\pi: M \rightarrow B$ be a principal $S^{1}$-bundle of Euler class e over a closed, connected, oriented manifold $B$ of dimension $2 n$. Suppose that $B$ admits a splitting $B=B_{+} \cup_{\Gamma} B_{-}$along a (possibly empty) codimension 1 submanifold $\Gamma$ such that there are symplectic forms $\omega_{ \pm}$on $\pm B_{ \pm}$and a cooriented contact structure $\operatorname{ker} \beta$ on $\Gamma$ satisfying conditions (i) and (ii) of Proposition 3.6 . Then $M$ admits an $S^{1}$-invariant contact structure with dividing set $\Gamma$.

Proof of Theorem 4.1 for $\Gamma=\emptyset$. If $\omega_{+}$is a symplectic form on $B$ with $-\left[\omega_{+} / 2 \pi\right]=$ $e$, take $\alpha$ to be a connection 1-form $\psi$ with curvature form $\omega_{+}$. Then we have $\alpha \wedge(\mathrm{d} \alpha)^{n}=\psi \wedge \pi^{*} \omega_{+}^{n}>0$. If $-B$ admits a symplectic form $\omega_{-}$with $\left[\omega_{-} / 2 \pi\right]=e$, set $\alpha=-\psi$, where $\psi$ is a connection 1 -form with curvature form $-\omega_{-}$. Then $\alpha \wedge(\mathrm{d} \alpha)^{n}=-\psi \wedge \pi^{*} \omega_{-}^{n}>0$.

From now on it will be assumed that $B$ decomposes as $B=B_{+} \cup_{\Gamma} B_{-}$with $\Gamma \neq \emptyset$. We begin by considering $B_{+}$and $B_{-}$separately. Our first aim is to modify $\omega_{ \pm}$in a neighbourhood of the boundary such that the new symplectic manifolds resemble strong fillings of $(\Gamma, \operatorname{ker} \beta)$. The next lemma mildly generalises an idea of Eliashberg [5], cf. 6].

Lemma 4.2. The symplectic forms $\omega_{ \pm}$can be modified in a collar neighbourhood of $\Gamma=\partial\left( \pm B_{ \pm}\right)$in $\pm B_{ \pm}$such that in a smaller collar neighbourhood $(-\varepsilon, 0] \times \Gamma$ we can write

$$
\omega_{ \pm}= \pm \omega_{ \pm}^{\Gamma}+\mathrm{d}\left(\mathrm{e}^{s} \beta\right)
$$

possibly after replacing $\beta$ by $K \beta$ for some large $K \in \mathbb{R}^{+}$. Here $\omega_{ \pm}^{\Gamma}$ are 2 -forms on $\Gamma$, pulled back to $(-\varepsilon, 0] \times \Gamma$ under the projection map to $\Gamma$.

Remark 4.3. For the proof we shall only assume that $\left( \pm B_{ \pm}, \omega_{ \pm}\right)$are weak fillings in the sense of $\left(\mathrm{w}_{2}\right)$. After the modification described in the lemma, and possibly after extending the new $\omega_{ \pm}$in the obvious way over an attached collar $[0, R] \times \Gamma$ for some large $R>0$, we obviously have a weak filling in the sense of $\left(\mathrm{w}_{1}\right)$. This proves Proposition 3.5 .

Proof of Lemma 4.2. For ease of notation we first consider $\left(B_{+}, \omega_{+}\right)$. Consider a tubular neighbourhood $[0,1] \times \Gamma$ of the boundary, where $\{1\} \times \Gamma \equiv \Gamma=\partial B_{+}$. Define the 2 -form $\omega_{+}^{\Gamma}$ on $[0,1] \times \Gamma$ by first restricting $\omega_{+}$to $T(\{0\} \times \Gamma)$ (i.e. pulling back under the inclusion $\{0\} \times \Gamma \subset[0,1] \times \Gamma)$ and then pulling back again to $[0,1] \times \Gamma$. Then both forms $\omega_{+}$and $\omega_{+}^{\Gamma}$ represent the cohomology class $-\left.2 \pi e\right|_{[0,1] \times \Gamma}$, so there is a 1 -form $\gamma$ on $[0,1] \times \Gamma$ such that

$$
\omega_{+}=\omega_{+}^{\Gamma}+\mathrm{d} \gamma
$$

We continue to write $\beta$ for the 1 -form on $[0,1] \times \Gamma$ obtained by pulling back the original $\beta$ from $\Gamma=\{1\} \times \Gamma$. Since $\left(B_{+}, \omega_{+}\right)$is a weak filling of $(\Gamma, \operatorname{ker} \beta)$, we may assume that the collar $[0,1] \times \Gamma$ had been chosen so small that condition $\left(\mathrm{w}_{2}\right)$ is satisfied on the tangent bundle $T(\{t\} \times \Gamma)$ for each $t \in[0,1]$, and for any convex linear combination of $\omega_{+}^{\Gamma}$ and $\omega_{+}$. In other words, for any $c_{t} \in[0,1]$ and function $b_{t}:\{t\} \times \Gamma \rightarrow \mathbb{R}_{0}^{+}$we have

$$
\beta \wedge\left(b_{t} \mathrm{~d} \beta+\omega_{+}^{\Gamma}+c_{t} \mathrm{~d} \gamma\right)^{n-1}>0 \text { on } T(\{t\} \times \Gamma) .
$$


In the sequel we shall take $b_{t}$ to be constant on $\{t\} \times \Gamma$, so we regard both $t \mapsto b_{t}$ and $t \mapsto c_{t}$ as real-valued functions on $[0,1]$. Set

$$
\tilde{\omega}_{+}=\omega_{+}^{\Gamma}+\mathrm{d}(c \gamma)+\mathrm{d}(b \beta)
$$

on $[0,1] \times \Gamma$, where the smooth functions $b$ and $c$ on $[0,1]$, are chosen as follows. Fix a small $\varepsilon>0$. Choose $b:[0,1] \rightarrow \mathbb{R}_{0}^{+}$monotonically increasing, identically 0 near $t=0$ and with $b^{\prime}(t)>0$ for $t>\varepsilon / 2$. Choose $c:[0,1] \rightarrow[0,1]$ identically 1 on $[0, \varepsilon]$ and identically 0 near $t=1$.

We compute

$$
\tilde{\omega}_{+}^{n}=\left(n \mathrm{~d} t \wedge\left(b^{\prime} \beta+c^{\prime} \gamma\right)+\left(b \mathrm{~d} \beta+\omega_{+}^{\Gamma}+c \mathrm{~d} \gamma\right)\right) \wedge\left(b \mathrm{~d} \beta+\omega_{+}^{\Gamma}+c \mathrm{~d} \gamma\right)^{n-1} .
$$

By our choices, the $2 n$-form

$$
\mathrm{d} t \wedge \beta \wedge\left(b \mathrm{~d} \beta+\omega_{+}^{\Gamma}+c \mathrm{~d} \gamma\right)^{n-1}
$$

is a volume form on $[0,1] \times \Gamma$, so is

$$
\left(\omega_{+}^{\Gamma}+c \mathrm{~d} \gamma\right)^{n}
$$

on $[0, \varepsilon] \times \Gamma$, where $c=1$, since $\omega_{+}^{\Gamma}+\mathrm{d} \gamma=\omega_{+}$. Thus, by choosing $b$ small on $[0, \varepsilon]$ and $b^{\prime}$ large compared with $\max \left\{1, b,\left|c^{\prime}\right|\right\}$ on $[\varepsilon, 1]$ (up to some multiplicative constants involving the norms of the differential forms in the above expression), one can ensure that $\tilde{\omega}_{+}^{n}>0$. Then $\tilde{\omega}_{+}$is a symplectic form on $[0,1] \times \Gamma$ and, in terms of the coordinate $s:=\log b(t)-\log b(1)$, this symplectic form looks like $\omega_{+}^{\Gamma}+\mathrm{d}\left(\mathrm{e}^{s} b(1) \beta\right)$ near $\{1\} \times \Gamma$.

For $\left(-B_{-}, \omega_{-}\right)$the argument is completely analogous, except that we take $\omega_{-}^{\Gamma}$ to be the restriction of $-\omega_{-}$to $T(\{0\} \times \Gamma)$. The value $b(1)$ may be chosen the same for $\omega_{+}$and $\omega_{-}$.

Remark 4.4. Our choice of sign in the preceding lemma implies that when we regard the 2 -forms $\omega_{ \pm}^{\Gamma}$ as forms on $\Gamma$, we have $-\left[\omega_{ \pm}^{\Gamma} / 2 \pi\right]=\left.e\right|_{\Gamma}$, so both forms $\omega_{ \pm}^{\Gamma}$ are curvature forms for the restriction of the $S^{1}$-bundle to $\Gamma$.

The following is a generalisation of the argument used for proving [8, Theorem 1].

Proof of Theorem 4.1 for $\Gamma \neq \emptyset$. By Lemma 4.2 we find a collar neighbourhood

$$
(-1-\varepsilon,-1] \times \Gamma
$$

of $\{-1\} \times \Gamma \equiv \Gamma=\partial B_{+}$in $B_{+}$where

$$
\omega_{+}=\omega_{+}^{\Gamma}+\mathrm{d}\left(\mathrm{e}^{t+1} \beta\right)
$$

the shift in the collar parameter is made for notational convenience below. Likewise, we have a collar neighbourhood

$$
[1,1+\varepsilon) \times \Gamma
$$

of $\{1\} \times \Gamma \equiv \Gamma=\partial\left(-B_{-}\right)$in $B_{-}$where

$$
\omega_{-}=-\omega_{-}^{\Gamma}+\mathrm{d}\left(\mathrm{e}^{-t+1} \beta\right)
$$

Write the base $B$ of the $S^{1}$-bundle as

$$
B_{+} \cup_{\Gamma}([-1,1] \times \Gamma) \cup_{\Gamma} B_{-} .
$$

Let $\psi_{ \pm}$be connection 1 -forms of the restriction of the $S^{1}$-bundle to $B_{ \pm}$with curvature forms $\pm \omega_{ \pm}$. Let $\psi_{ \pm}^{\Gamma}$ be connection 1 -forms of the $S^{1}$-bundle over $\Gamma$ with curvature form $\omega_{ \pm}^{\Gamma}$. 
Lemma 4.5. The choices in the preceding argument can be made in such a way that over the two collars $(-1-\varepsilon,-1] \times \Gamma$ and $[1,1+\varepsilon) \times \Gamma$ we have

$$
\psi_{ \pm}=\psi_{ \pm}^{\Gamma} \pm \mathrm{e}^{ \pm t+1} \beta
$$

respectively, perhaps at the cost of taking a slightly smaller $\varepsilon>0$.

Proof. We only deal with $\psi_{+}$; the argument for $\psi_{-}$is completely analogous. Over the collar $(-1-\varepsilon,-1] \times \Gamma$ the connection forms $\psi_{+}$and $\psi_{+}^{\Gamma}+\mathrm{e}^{t+1} \beta$ have the same curvature form $\omega_{+}$. It follows that

$$
\psi_{+}=\psi_{+}^{\Gamma}+\mathrm{e}^{t+1} \beta+\gamma
$$

with $\gamma$ a closed horizontal 1-form. Choose a closed 1-form $\gamma^{\Gamma}$ on $\Gamma$, which we also interpret as a 1 -form on $(-1-\varepsilon,-1] \times \Gamma$, representing the same class as $\gamma$ in $H_{\mathrm{dR}}^{1}((-1-\varepsilon,-1] \times \Gamma)$. Then we can write

$$
\gamma=\gamma^{\Gamma}+\mathrm{d} h
$$

for some smooth function on $(-1-\varepsilon,-1] \times \Gamma$. Replace $\psi_{+}^{\Gamma}$ by $\psi_{+}^{\Gamma}+\gamma^{\Gamma}$, and $\psi_{+}$by $\psi_{+}-\mathrm{d}(\chi h)$, where $\chi:(-1-\varepsilon,-1] \rightarrow[0,1]$ interpolates smoothly between 0 near $-1-\varepsilon$ and 1 near -1 . Then the new $\psi_{+}$still extends as before over $B_{+}$, and near $\{-1\} \times \Gamma$ we have the equality claimed in the lemma.

We continue with the proof of Theorem 4.1, Let $\psi_{t}^{\Gamma}, t \in[-1,1]$, be a smooth family of connection 1-forms on the $S^{1}$-bundle over $\Gamma$ with $\psi_{t}^{\Gamma}=\psi_{ \pm}^{\Gamma}$ for $t$ near $\mp 1$.

Now choose two smooth functions $f$ and $g$ on the interval $(-1-\varepsilon, 1+\varepsilon)$ subject to the following conditions (see Figure 1):

- $f$ is an even and nowhere zero function with $f(t)=\mathrm{e}^{t+1}$ near $(-1-\varepsilon,-1]$,

- $g$ is an odd function with $g(t)=1$ near $(-1-\varepsilon,-1]$ and a single zero at 0 ,

- $f^{\prime} g-f g^{\prime}>0$,

- $f \gg 1$ and $f^{\prime} g-f g^{\prime} \gg 1$ where $g^{\prime} \neq 0$.
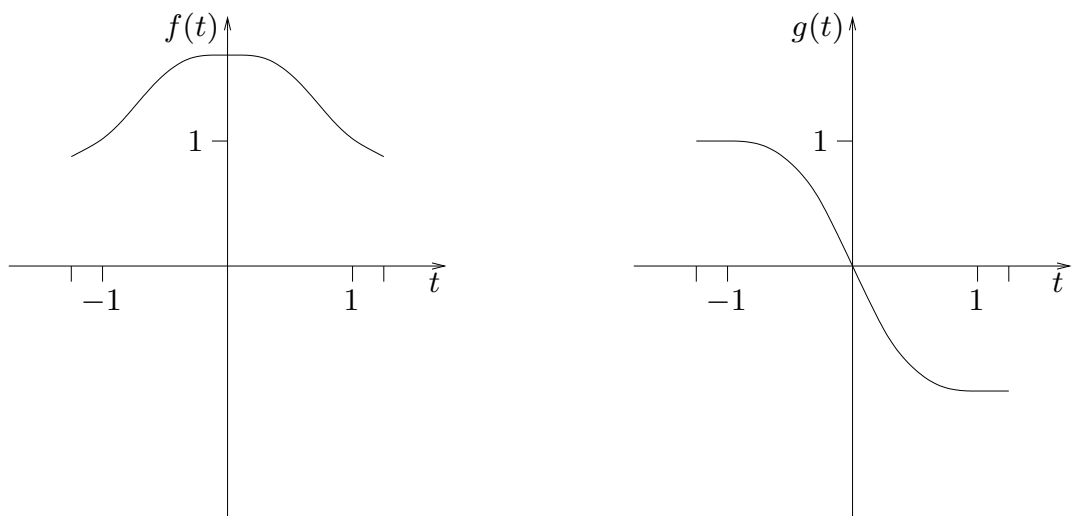

Figure 1. The functions $f$ and $g$.

Define a smooth $S^{1}$-invariant 1 -form $\alpha$ on the $S^{1}$-bundle over $B$ by

$$
\alpha= \begin{cases}\psi_{+} & \text {over } B_{+}, \\ f \beta+g \psi_{t}^{\Gamma} & \text { over }[-1,1] \times \Gamma, \\ -\psi_{-} & \text {over } B_{-} .\end{cases}
$$


Over $B_{ \pm}$this defines a contact form by the same computation as in the proof for the case $\Gamma=\emptyset$. Over $[-1,1] \times \Gamma$ we compute

$$
\begin{aligned}
\alpha \wedge(\mathrm{d} \alpha)^{n}= & \left(f \beta+g \psi_{t}^{\Gamma}\right) \wedge n\left(f \mathrm{~d} \beta+g \mathrm{~d} \psi_{t}^{\Gamma}\right)^{n-1} \wedge \mathrm{d} t \\
& \wedge\left(f^{\prime} \beta+g^{\prime} \psi_{t}^{\Gamma}+g\left(\partial \psi_{t}^{\Gamma} / \partial t\right)\right) \\
= & n \psi_{t}^{\Gamma} \wedge \mathrm{d} t \wedge\left(\left(f^{\prime} g-f g^{\prime}\right) \beta+g^{2}\left(\partial \psi_{t}^{\Gamma} / \partial t\right)\right) \\
& \wedge\left(f \mathrm{~d} \beta+g \mathrm{~d} \psi_{t}^{\Gamma}\right)^{n-1} .
\end{aligned}
$$

Notice that $\partial \psi_{t}^{\Gamma} / \partial t$ is a horizontal 1-form, so the term where we wedge this with $\beta$ rather than $\psi_{t}^{\Gamma}$ from the first factor yields a horizontal $(2 n+1)$-form, i.e. zero.

Near $t=-1$ we have $g \equiv 1$ and $\psi_{t}^{\Gamma} \equiv \psi_{+}^{\Gamma}$, hence $\mathrm{d} \psi_{t}^{\Gamma} \equiv \omega_{+}^{\Gamma}$. With condition (ii) from Proposition 3.6 this implies

$$
\alpha \wedge(\mathrm{d} \alpha)^{n}=n f^{\prime} \psi_{+}^{\Gamma} \wedge \mathrm{d} t \wedge \beta \wedge\left(f \mathrm{~d} \beta+\omega_{+}^{\Gamma}\right)^{n-1}>0 .
$$

Near $t=1$ we have $g \equiv-1$ and $\psi_{t}^{\Gamma} \equiv \psi_{-}^{\Gamma}$, hence $\mathrm{d} \psi_{t}^{\Gamma} \equiv \omega_{-}^{\Gamma}$. Recall that $\omega_{-}^{\Gamma}$ was defined as the restriction of $-\omega_{-}$, so we get

$$
\alpha \wedge(\mathrm{d} \alpha)^{n}=-n f^{\prime} \psi_{-}^{\Gamma} \wedge \mathrm{d} t \wedge \beta \wedge\left(f \mathrm{~d} \beta-\omega_{-}^{\Gamma}\right)^{n-1}>0 .
$$

Finally, over the region where $g^{\prime} \neq 0$, we have $f \gg 1$ and $f^{\prime} g-f g^{\prime} \gg 1$. It follows that the positive summand

$$
n f^{n-1}\left(f^{\prime} g-f g^{\prime}\right) \psi_{t}^{\Gamma} \wedge \mathrm{d} t \wedge \beta \wedge(\mathrm{d} \beta)^{n-1}
$$

in the expression for $\alpha \wedge(\mathrm{d} \alpha)^{n}$ will dominate all other summands.

The dividing set of the contact structure ker $\alpha$ coincides with the zero set $\{0\} \times \Gamma$ of $g$. This completes the proof of the theorem.

\section{EXAMPLES}

5.1. From trivial to nontrivial bundles. Here is a simple corollary of our main theorem.

Corollary 5.1. Let $B$ be a closed, oriented manifold of dimension $2 n$. If the trivial $S^{1}$-bundle over $B$ admits an $S^{1}$-invariant contact structure with dividing set $\Gamma$, then so do all $S^{1}$-bundles over $B$.

Proof. If the trivial $S^{1}$-bundle over $B$ admits an $S^{1}$-invariant contact structure with dividing set $\Gamma$, then by Proposition 3.6 the base $B$ has a splitting $B=B_{+} \cup_{\Gamma} B_{-}$ with a contact structure $\operatorname{ker} \beta$ on $\Gamma$ and exact symplectic forms $\mathrm{d} \lambda_{ \pm}$on $\pm B_{ \pm}$such that $\left( \pm B_{ \pm}, \mathrm{d} \lambda_{ \pm}\right)$are weak fillings of $(\Gamma, \operatorname{ker} \beta)$.

Given an $S^{1}$-bundle over $B$ of Euler class $e \in H_{\mathrm{dR}}^{2}(B)$, choose 2-forms $\sigma_{ \pm}$on $B_{ \pm}$with $\mp\left[\sigma_{ \pm} / 2 \pi\right]=\left.e\right|_{B_{ \pm}}$. For $K \in \mathbb{R}^{+}$sufficiently large, the 2-forms $\omega_{ \pm}:=$ $\sigma_{ \pm}+K \mathrm{~d} \lambda_{ \pm}$are symplectic forms satisfying conditions (i) and (ii) of Proposition 3.6. Then the result follows from Theorem 4.1.

Using a result of Baykur [1] on decompositions of 4-manifolds, it was shown in 8, Corollary 2] that the trivial $S^{1}$-bundle over any closed, oriented 4-manifold admits an $S^{1}$-invariant contact structure. So the next corollary is immediate. We give a direct proof illuminating the role of Baykur's result.

Corollary 5.2. Any $S^{1}$-bundle over any closed, oriented 4-manifold admits an $S^{1}$-invariant contact structure. 
Proof. According to [1, Theorem 5.2], any closed, oriented 4-manifold $B$ admits a splitting $B=B_{+} \cup_{\Gamma} B_{-}$with exact symplectic forms $\mathrm{d} \lambda_{ \pm}$on $\pm B_{ \pm}$that provide a filling of one and the same contact structure on $\Gamma$. In fact, the $\left( \pm B_{ \pm}, \mathrm{d} \lambda_{ \pm}\right)$can be taken to be Stein fillings.

Then the argument concludes as in the proof of the preceding corollary.

In [8] it was also shown that $\mathbb{C P}^{2} \times S^{1}$ admits a contact structure in every homotopy class of almost contact structures (i.e. reduction of the structure group to $\mathrm{U}(2) \times 1)$. The same argument as in the proof of Corollary 5.1 allows us to extend this to nontrivial bundles: on any given $S^{1}$-bundle over $\mathbb{C P}^{2}$, any homotopy class of $S^{1}$-invariant almost contact structures contains a contact structure.

5.2. It's all in the twist. With the help of an example we want to illustrate that the topological type of the total space $M$ of an $S^{1}$-bundle with an invariant contact structure is not, in general, determined by the symplectic splitting of the base $B$. This happens whenever there are nontrivial cohomology classes in $H_{\mathrm{dR}}^{2}(B)$ that restrict to zero on the pieces $B_{ \pm}$of the splitting, or in the presence of torsion in $H^{2}(B ; \mathbb{Z})$.

We take $B=T^{2} \times S^{2}$ and split it into $B_{ \pm}=T^{2} \times D_{ \pm}^{2}$. Write $\theta_{1}, \theta_{2}$ for circle coordinates on $T^{2}$, and $x, y$ (resp. $r, \varphi$ ) for Cartesian (resp. polar) coordinates on $\pm D_{ \pm}^{2}$. Then the gluing $D_{+}^{2} \cup_{S^{1}} D_{-}^{2}$ is given by $\varphi \mapsto \varphi$.

On $\pm B_{ \pm}$we have the symplectic form $\mathrm{d} x \wedge \mathrm{d} \theta_{1}-\mathrm{d} y \wedge \mathrm{d} \theta_{2}$. This admits the Liouville vector field $x \partial_{x}+y \partial_{y}$, and hence gives a strong filling of $\Gamma=T^{3}=\partial\left( \pm B_{ \pm}\right)$ with contact structure $\operatorname{ker}\left(\cos \varphi \mathrm{d} \theta_{1}-\sin \varphi \mathrm{d} \theta_{2}\right)$.

For any $k \in \mathbb{Z}$, the two trivial bundles $B_{ \pm} \times S_{\theta}^{1}$ can be glued using the map

$$
g_{k}:\left(\theta_{1}, \theta_{2}, \varphi, \theta\right) \longmapsto\left(\theta_{1}, \theta_{2}, \varphi, \theta-k \varphi\right)
$$

on the boundary. The Euler class $e \in H_{\mathrm{dR}}^{2}(B)$ of the resulting $S^{1}$-bundle over $B$ satisfies $\left\langle e,\left[T^{2} \times *\right]\right\rangle=0$ and $\left\langle e,\left[* \times S^{2}\right]\right\rangle=k$. The restriction of $e$ to $B_{ \pm}$is the zero class.

According to Theorem 4.1, each of these bundles over $B$ carries an $S^{1}$-invariant contact structure that induces the same decomposition of $B$ into two exact symplectic pieces $\pm B_{ \pm}$. We shall return to this example in Section 6 ,

5.3. Open books and the Bourgeois construction. An open book decomposition of a manifold $N$ consists of a codimension 2 submanifold $B_{N}$, called the binding, and a (smooth, locally trivial) fibration $\mathfrak{p}: N \backslash B_{N} \rightarrow S^{1}$. The closures of the fibres $\mathfrak{p}^{-1}(\varphi), \varphi \in S^{1}$, are called the pages. Moreover, it is required that the binding $B_{N}$ have a trivial tubular neighbourhood $B_{N} \times D^{2}$ in which $\mathfrak{p}$ is given by the angular coordinate in the $D^{2}$-factor.

If $N$ and $B_{N}$ are oriented, we orient the pages $p^{-1}(\varphi)$ consistently with their boundary $B_{N}$. This is the same as saying that $\partial_{\varphi}$ together with the orientation of the page gives the orientation of $N$, cf. [7. p. 154].

Following Giroux [11, we say that a contact structure $\xi=\operatorname{ker} \alpha$ on $N$ defined by a positive contact form $\alpha$ is supported by the open book decomposition $\left(B_{N}, \mathfrak{p}\right)$ if

(i) the 2-form $\mathrm{d} \alpha$ induces a positive symplectic form on each fibre of $\mathfrak{p}$, and

(ii) the 1-form $\alpha$ induces a positive contact form on $B_{N}$.

Giroux has shown that every contact structure on a closed manifold is supported by an open book. This fact was used by Bourgeois 4 to show that, starting from a 
contact structure on a closed manifold $N$, one can produce a $T^{2}$-invariant contact structure on the product of $N$ with a 2-torus $T^{2}$. In particular, all odd-dimensional tori admit a contact structure.

We now want to indicate briefly how the Bourgeois construction can be interpreted in the framework of the present note. Thus, let $N$ be a closed, connected manifold of dimension $2 n-1 \geq 3$ with a contact structure $\xi=\operatorname{ker} \alpha$ supported by an open book decomposition $\left(B_{N}, \mathfrak{p}\right)$. Let $(r, \varphi)$ be polar coordinates on the $D^{2}$ factor of a neighbourhood $B_{N} \times D^{2}$ of the binding $B_{N}$, such that $\mathfrak{p}: N \backslash B_{N} \rightarrow S^{1}$ is given by $\varphi$ in that neighbourhood. We choose this neighbourhood so small that $\alpha$ restricts to a contact form on the manifold $B_{N} \times\{z\}$ for any $z \in D^{2}$.

Choose a smooth function $\rho$ of the variable $r$ on $B_{N} \times D^{2}$ satisfying the requirements that

- $\rho(r)=r$ near $B_{N} \equiv B_{N} \times\{0\}$,

- $\rho^{\prime}(r) \geq 0$,

- $\rho \equiv 1$ near $B_{N} \times \partial D^{2}$.

Extend $\rho$ to a smooth function on $N$ by setting it equal to 1 outside $B_{N} \times D^{2}$. Then $\mathrm{x}:=\rho \cos \varphi$ and $\mathrm{y}:=\rho \sin \varphi$ are smooth functions on $N$ that coincide with the Cartesian coordinate functions $x, y$ on the $D^{2}$-factor near $B_{N} \times\{0\} \subset B_{N} \times D^{2}$. The identity

$$
\mathrm{xdy}-\mathrm{ydx}=\rho^{2} \mathrm{~d} \varphi
$$

holds on all of $N$.

In [4, cf. [7, Theorem 7.3.6], it was shown that

$$
\alpha-\mathrm{xd} \phi+\mathrm{yd} \theta
$$

defines a $T^{2}$-invariant contact structure on $N \times T^{2}=N \times S_{\phi}^{1} \times S_{\theta}^{1}$. Our Proposition 3.6 then tells us that the 1-form

$$
\beta_{0}:=\alpha-\mathrm{xd} \phi
$$

is an $S^{1}$-invariant contact form on

$$
\Gamma:=\{\mathrm{y}=0\} \times S_{\phi}^{1}=\left(\left(-\mathfrak{p}^{-1}(0)\right) \cup_{B_{N}} \mathfrak{p}^{-1}(\pi)\right) \times S_{\phi}^{1},
$$

and the 2-form

$$
\omega_{ \pm}:= \pm \mathrm{d}\left(\frac{\alpha}{\mathrm{y}}-\frac{\mathrm{x}}{\mathrm{y}} \mathrm{d} \phi\right)
$$

is an $S^{1}$-invariant symplectic form on $\operatorname{Int}\left( \pm B_{ \pm}\right)$, where

$$
B_{+}:=\mathfrak{p}^{-1}([0, \pi]) \times S_{\phi}^{1}, \quad B_{-}:=\mathfrak{p}^{-1}([\pi, 2 \pi]) \times S_{\phi}^{1},
$$

i.e. $B_{ \pm}=\{ \pm \mathrm{y} \geq 0\} \times S_{\phi}^{1}$. Conversely, one can check these properties directly and thus derive Bourgeois's result.

Notice that the $S^{1}$-invariant contact structure on $\Gamma$ corresponds to the splitting of $\{\mathrm{y}=0\}$ along the hypersurface $\{\mathrm{x}=0=\mathrm{y}\}$. The splitting of $N \times S_{\phi}^{1}$ into two exact symplectic pieces $\pm B_{ \pm}$comes from taking the two 'halves' of the open book on $N$, crossed with $S_{\phi}^{1}$. This allows one to give simple explicit symplectic splittings of manifolds of the form $N \times S^{1}$.

With Corollary 5.1 we obtain the following generalisation of Bourgeois's result.

Corollary 5.3. Let $N$ be a closed manifold admitting a contact structure. Then any principal $S^{1}$-bundle over $N \times S^{1}$ admits an $S^{1}$-invariant contact structure. 


\section{IdEAL LiOUVille DOMAins}

The manuscript of 14 became available only after the first version of the present note had been completed. In this section, which is based on correspondence with Patrick Massot, we wish to explain how our construction can be phrased in the more sophisticated language of that paper.

The following definition, taken from [14, Section 4.2], is due to Giroux.

Definition 6.1 (Giroux). An ideal Liouville domain is a triple $\left(\Sigma, \omega_{\text {Int }}, \eta\right)$ consisting of a compact oriented $2 n$-manifold $\Sigma$ with boundary, a symplectic form $\omega_{\text {Int }}$ on the interior $\operatorname{Int}(\Sigma)$, and a contact structure $\eta$ on the boundary $\partial \Sigma$, such that there is an auxiliary 1 -form $\lambda$ on $\operatorname{Int}(\Sigma)$ with the following properties:

(i) $\mathrm{d} \lambda=\omega_{\text {Int }}$;

(ii) for some (and hence any) smooth function $u: \Sigma \rightarrow \mathbb{R}_{0}^{+}$with $\partial \Sigma$ as its regular zero set, the 1 -form $\beta:=u \lambda$ on $\operatorname{Int}(\Sigma)$ extends to $\partial \Sigma$ as a contact form for $\eta$.

Given an ideal Liouville domain $\left(\Sigma, \omega_{\text {Int }}, \eta\right)$, it is easy to check that the 1 -form $u \lambda+u \mathrm{~d} \theta$ defines an $S^{1}$-invariant contact structure $\xi$ on $\Sigma \times S^{1}$. In 14, the pair $\left(\Sigma \times S^{1}, \xi\right)$ is called the Giroux domain associated with $\left(\Sigma, \omega_{\text {Int }}, \eta\right)$.

We now adapt these definitions to nontrivial $S^{1}$-bundles.

Definition 6.2. Let $\Sigma$ and $\eta$ be as above, $\omega_{\text {Int }}$ a symplectic form on $\operatorname{Int}(\Sigma)$, and $c$ a cohomology class in $H^{2}(\Sigma ; \mathbb{Z})$. The tuple $\left(\Sigma, \omega_{\text {Int }}, \eta, c\right)$ is called an ideal Liouville domain if for some (and hence any) closed 2 -form $\omega$ on $\Sigma$ with $-[\omega / 2 \pi]=c \otimes \mathbb{R} \in$ $H_{\mathrm{dR}}^{2}(\Sigma)$ there exists a 1 -form $\lambda$ on $\operatorname{Int}(\Sigma)$ such that $\mathrm{d} \lambda=\omega_{\text {Int }}-\omega$ and condition (ii) holds as before.

The choice of $\omega$ is indeed irrelevant. If $\omega$ is replaced by $\omega^{\prime}$ with $\left[\omega^{\prime}\right]=[\omega]$, then $\omega^{\prime}=\omega+\mathrm{d} \mu$ with $\mu$ a 1 -form on $\Sigma$. So we simply need to replace $\lambda$ by $\lambda^{\prime}=\lambda-\mu$. Since $\mu$ is defined on $\Sigma$ (including the boundary), the extension of $u \lambda^{\prime}$ to $\partial \Sigma$ coincides with that of $u \lambda$. Observe that an ideal Liouville domain $\left(\Sigma, \omega_{\text {Int }}, \eta, 0\right)$ is an ideal Liouville domain in the sense of Giroux.

Now let $\pi: M \rightarrow \Sigma$ be the principal $S^{1}$-bundle over $\Sigma$ of (integral) Euler class $c$. Choose a connection 1-form $\psi$ with curvature form $\omega$ on this bundle. As before, it is easy to check that the 1 -form $u \psi+u \gamma$ defines a contact structure $\xi$ on $M$. Notice that $\xi$ intersects $\pi^{-1}(\partial \Sigma)$ transversely; the intersection $\xi \cap T\left(\pi^{-1}(\partial \Sigma)\right)$ is the tangent hyperplane field given by the kernel of (the lift of) $\beta$. We call $(M, \xi)$ the contactisation of $\left(\Sigma, \omega_{\text {Int }}, \eta, c\right)$.

Definition 6.3. Let $B$ be a closed, oriented manifold of dimension $2 n$. An ideal Liouville splitting of class $c \in H^{2}(B ; \mathbb{Z})$ is a decomposition $B=B_{+} \cup_{\Gamma} B_{-}$along a two-sided (but not necessarily connected) hypersurface $\Gamma$, oriented as the boundary of $B_{+}$, together with a contact structure $\eta$ on $\Gamma$ and symplectic forms $\omega_{ \pm}$on $\operatorname{Int}\left( \pm B_{ \pm}\right)$, such that

$$
\left( \pm B_{ \pm}, \omega_{ \pm}, \eta, \pm\left. c\right|_{B_{ \pm}}\right)
$$

are ideal Liouville domains.

In this terminology, Proposition 3.6 can be read as saying that an $S^{1}$-invariant contact structure $\xi=\operatorname{ker}(\beta+u \psi)$ on the principal $S^{1}$-bundle $M \rightarrow B$ defined by $c \in H^{2}(B ; \mathbb{Z})$ leads to a Liouville splitting of $B$ of class $c$. It will become apparent 
presently why we need to fix an integral class $c$ in our notion of ideal Liouville splitting.

Conversely, assume that the base $B$ of the $S^{1}$-bundle $M \rightarrow B$ of Euler class $c \in$ $H^{2}(B ; \mathbb{Z})$ admits an ideal Liouville splitting of class $c$. The contactisations $\left(M_{ \pm}, \xi_{ \pm}\right)$ of $B_{ \pm}$induce the same non-singular and $S^{1}$-invariant characteristic distribution $\xi_{ \pm} \cap T\left(\pi^{-1}(\Gamma)\right)$. Therefore, any equivariant diffeomorphism of $\pi^{-1}(\Gamma)$, in particular the one necessary to recover $M$, can be used to obtain a contact manifold by gluing these two contactisations. This is true thanks to the following proposition, which is folklore.

Proposition 6.4. Let $H$ be a closed hypersurface in a contact manifold $(M, \xi=$ $\operatorname{ker} \alpha)$. Then the germ of $\xi$ near $H$ is determined by the 1 -form $\left.\alpha\right|_{T H}$. In particular, if $\left.\xi\right|_{H}$ is transverse to $H$, then the germ of $\xi$ near $H$ is determined by the codimension 1 distribution $T H \cap \xi$ on $H$.

Proof. By passing to a double cover, if necessary, we may assume that $H$ is orientable. Let $\xi_{0}=\operatorname{ker} \alpha_{0}$ and $\xi_{1}=\operatorname{ker} \alpha_{1}$ be two contact structures near $H$ with $\left.\alpha_{0}\right|_{T H}=\left.\alpha_{1}\right|_{T H}$. Identify a neighbourhood of $H$ with $H \times \mathbb{R}$. Then we can write $\alpha_{i}=\beta_{i}^{r}+u_{i}^{r} \mathrm{~d} r, i=0,1$, where $\beta_{i}^{r}, r \in \mathbb{R}$, is a smooth family of 1 -forms on $H$, and $u_{i}^{r}: H \rightarrow \mathbb{R}$ a smooth family of functions. By assumption we have $\beta_{0}^{0}=\beta_{1}^{0}$ and hence $\mathrm{d} \beta_{0}^{0}=\mathrm{d} \beta_{1}^{0}$. The contact condition for the $\alpha_{i}$ looks as follows, where we drop the subscript $i$ for the moment (and $\operatorname{dim} M=2 n+1$ ):

$$
\left(-n \beta^{r} \wedge\left(\partial \beta^{r} / \partial r\right)+n \beta^{r} \wedge \mathrm{d} u^{r}+u^{r} \mathrm{~d} \beta^{r}\right) \wedge\left(\mathrm{d} \beta^{r}\right)^{n-1} \wedge \mathrm{d} r>0 .
$$

This expression is linear in $\partial \beta^{r} / \partial r$ and $u^{r}$. It follows that on a neighbourhood of $H$ the convex linear interpolation $\alpha_{t}=(1-t) \alpha_{0}+t \alpha_{1}$ is a contact form for all $t \in[0,1]$.

Now apply the Moser trick to this family, cf. [7, Chapter 2]. One finds that $\xi_{0}$ is isotopic to $\xi_{1}$ via an isotopy $\psi_{t}$ given as the flow of a vector field $X_{t} \in \operatorname{ker} \alpha_{t}$ determined by the equation

$$
\dot{\alpha}_{t}+i_{X_{t}} \mathrm{~d} \alpha_{t}=\mu_{t} \alpha_{t},
$$

where the function $\mu_{t}$ is given by evaluating $\dot{\alpha}_{t}$ on the Reeb vector field of $\alpha_{t}$.

On $T H$ we have $\dot{\alpha}_{t}=0$. So for $\mathbf{v} \in T H \cap \xi_{i}$ we have $\mathrm{d} \alpha_{t}\left(X_{t}, \mathbf{v}\right)=0$. This implies that $\left.X_{t}\right|_{H} \in T H \cap \xi_{i}$, i.e. the flow of $X_{t}$ preserves both $H$ and the characteristic distribution. Since $H$ is closed, $X_{t}$ integrates to a flow $\psi_{t}, t \in[0,1]$, near $H$.

In the present situation all forms in question are $S^{1}$-invariant. Then so will be the isotopy $\psi_{t}$. This makes the gluing $S^{1}$-equivariant.

Beware that, as in the example in Section 5.2, there may be a choice of gluing that can affect the global topology. Indeed, along two boundary components with the same characteristic distribution one can glue using any $S^{1}$-equivariant diffeomorphism that preserves this distribution.

Let us consider that example in a little more detail. The manifold $T^{2} \times D^{2}$ becomes an ideal Liouville domain (in the narrow sense, i.e. with $c=0$ ) by setting

$$
\omega_{\text {Int }}=\mathrm{d}\left(\frac{x}{1-r^{2}} \mathrm{~d} \theta_{1}-\frac{y}{1-r^{2}} \mathrm{~d} \theta_{2}\right)
$$

and $\eta=\operatorname{ker}\left(\cos \varphi \mathrm{d} \theta_{1}-\sin \varphi \mathrm{d} \theta_{2}\right)$. The gluing maps $g_{k}$ preserve the characteristic distribution induced on $T^{2} \times S^{1} \times S^{1}$ by the contactisation, and hence can be used to glue two such contactisations. 
Thus, we see that an ideal Liouville splitting without a prescribed class $c \in$ $H^{2}(B ; \mathbb{Z})$ may not lead to a unique $(M, \xi)$. If there is torsion in $H^{2}\left(B_{ \pm} ; \mathbb{Z}\right)$, the class $e=c \otimes \mathbb{R}$ does not determine the topology of the contactisations; if there is a class in $H^{2}(B ; \mathbb{Z})$ that restricts to zero on $B_{ \pm}$, the topology of the manifold obtained by gluing the two contactisations may not be determined by $\left.c\right|_{B_{ \pm}}$.

The following theorem generalises the equivariant classification result of Lutz [13].

Theorem 6.5. Let $\pi: M \rightarrow B$ be the $S^{1}$-bundle of Euler class $c \in H^{2}(B ; \mathbb{Z})$. Two invariant contact structures on $M$ are equivariantly diffeomorphic if and only if they induce diffeomorphic Liouville splittings of $B$ of class $c$, where it is understood that the diffeomorphism of $B$ preserves the class $c$.

Proof. We consider the case $\Gamma \neq \emptyset$. All arguments go through for the case $\Gamma=\emptyset$, i.e. $B=B_{+}$or $B=B_{-}$, with the obvious modifications.

For the 'only if' direction we first need to check that the Liouville splitting induced by an invariant contact structure does not depend on choices. For notational convenience we only consider $B_{+}$.

Given a connection 1-form $\psi$ on $M$ with curvature 2-form $\omega$, and an invariant contact form $\alpha=\beta+u \psi$, we get the ideal Liouville domain $\left(B_{+}, \omega_{+}, \eta,\left.c\right|_{B_{+}}\right)$, where

$$
\omega_{+}=\left.(\mathrm{d}(\beta / u)+\omega)\right|_{\operatorname{Int}\left(B_{+}\right)}
$$

and $\eta=\operatorname{ker}\left(\left.\beta\right|_{T \Gamma}\right)$. If the connection 1-form is replaced by $\psi^{\prime}=\psi+\gamma$, with $\gamma$ a 1 -form lifted from $B$, then $\beta$ needs to be replaced by $\beta^{\prime}=\beta-u \gamma$, and $\omega$ is replaced by $\omega^{\prime}=\omega+\mathrm{d} \gamma$. The symplectic form $\omega_{+}$remains unchanged. If the contact form $\alpha$ is replaced by $f \alpha$ for some invariant function $f: M \rightarrow \mathbb{R}^{+}$, then $\beta^{\prime}=f \beta$ and $u^{\prime}=f u$, so again $\omega_{+}$does not change. Also, these choices do not affect the contact structure $\eta$ on $\Gamma$.

An equivariant diffeomorphism of invariant contact structures on $M$ clearly induces a diffeomorphism of the induced ideal Liouville splittings. Since the $S^{1}$-bundle is determined by the class $c \in H^{2}(B ; \mathbb{Z})$, the induced diffeomorphism of $B$ preserves this class.

Conversely, i.e. for the 'if' direction, suppose that $\alpha$ and $\alpha^{\prime}$ are invariant contact forms on $M$ inducing Liouville splittings (of class $c$ ) of $B$ that are diffeomorphic via some diffeomorphism $\phi: B \rightarrow B$. Write $\phi^{*} M$ for the total space of the $S^{1}$-bundle over $B$ obtained by pulling back the bundle $M \rightarrow B$. There is an equivariant diffeomorphism $\tilde{\phi}: \phi^{*} M \rightarrow M$ covering $\phi$, and $\tilde{\phi}^{*} \alpha^{\prime}$ induces the same ideal Liouville splitting as $\alpha$.

Since $\phi$ preserves the class $c$ in integral cohomology, the bundle $\phi^{*} M \rightarrow B$ is bundle isomorphic to $M \rightarrow B$, i.e. there is an equivariant diffeomorphism $M \rightarrow$ $\phi^{*} M$ over the identity. So we may regard $\phi^{*} \alpha^{\prime}$ as an invariant contact form on $M$, defining the same ideal Liouville splitting as $\alpha$.

Write $\alpha=\beta+u \psi$. Then without loss of generality we can write $\phi^{*} \alpha^{\prime}=\beta^{\prime}+u \psi$. Both contact forms induce the same symplectic form $\omega_{+}$on $\operatorname{Int}\left(B_{+}\right)$, hence

$$
\left.\mathrm{d}(\beta / u)\right|_{\operatorname{Int}\left(B_{+}\right)}=\left.\mathrm{d}\left(\beta^{\prime} / u\right)\right|_{\operatorname{Int}\left(B_{+}\right)} .
$$

Moreover, $\beta$ and $\beta^{\prime}$ induce the same contact structure $\eta$ on $\Gamma$, i.e.

$$
\operatorname{ker}\left(\left.\beta\right|_{T \Gamma}\right)=\operatorname{ker}\left(\left.\beta^{\prime}\right|_{T \Gamma}\right) .
$$

Recall from Section 3 that $\alpha \wedge(\mathrm{d} \alpha)^{n}=\psi \wedge \Omega$, with

$$
\left.\Omega\right|_{\operatorname{Int}\left(B_{+}\right)}=u^{n+1}(\mathrm{~d}(\beta / u)+\omega)^{n}
$$


and

$$
\left.\Omega\right|_{\Gamma}=-n \mathrm{~d} u \wedge \beta \wedge(\mathrm{d} \beta)^{n-1} .
$$

It follows that $(1-t) \beta+t \beta^{\prime}+u \psi$ is a contact form for all $t \in[0,1]$. Gray stability then yields an $S^{1}$-equivariant contact isotopy between $\operatorname{ker} \alpha$ and $\operatorname{ker} \phi^{*} \alpha^{\prime}$.

The proof of Corollary 5.1 can likewise be phrased in terms of ideal Liouville domains.

Acknowledgement. We are grateful to Patrick Massot for explaining to us how to interpret our result in the language of ideal Liouville domains. We thank the referee for constructive comments on an earlier version of this paper.

\section{REFERENCES}

[1] R. İ. BAYKuR, 'Kähler decompositions of 4-manifolds', Algebr. Geom. Topol. 6 (2006) 12391265.

[2] W. M. Boоthby And H. C. WAng, 'On contact manifolds', Ann. of Math. (2) 68 (1958) $721-734$.

[3] R. Bott and L. W. Tu, Differential Forms in Algebraic Topology, Graduate Texts in Mathematics 82 (Springer-Verlag, Berlin, 1982).

[4] F. Bourgeois, 'Odd dimensional tori are contact manifolds', Int. Math. Res. Not. 2002 (2002) $1571-1574$.

[5] Ya. Eliashberg, 'On symplectic manifolds with some contact properties', J. Differential Geom. 33 (1991) 233-238.

[6] H. Geiges, 'Contact Dehn surgery, symplectic fillings, and property P for knots', Expo. Math. 24 (2006) 273-280.

[7] H. Geiges, An Introduction to Contact Topology, Cambridge Studies in Advanced Mathematics 109 (Cambridge University Press, Cambridge, 2008).

[8] H. Geiges And A. I. Stipsicz, 'Contact structures on product five-manifolds and fibre sums along circles', Math. Ann. 348 (2010) 195-210.

[9] E. Giroux, 'Convexité en topologie de contact', Comment. Math. Helv. 66 (1991) 637-677.

[10] E. Giroux, 'Structures de contact sur les variétés fibrées en cercles au-dessus d'une surface, Comment. Math. Helv. 76 (2001), 218-262.

[11] E. Giroux, 'Géométrie de contact: de la dimension trois vers les dimensions supérieures', Proceedings of the International Congress of Mathematicians, Vol. II (Higher Education Press, Beijing, 2002) 405-414.

[12] S. KoBAyASHI, 'Principal fibre bundles with the 1-dimensional toroidal group', Tôhoku Math. J. (2) 8 (1956) 29-45.

[13] R. Lutz, 'Structures de contact sur les fibrés principaux en cercles de dimension trois', Ann. Inst. Fourier (Grenoble) 27 (1977) no. 3, 1-15.

[14] P. Massot, K. Niederkrüger and C. Wende, 'Weak and strong fillability of higherdimensional contact manifolds', preprint (2011), arXiv: 1111.6008.

Department of Mathematics, Peking University, Beijing 100871, P. R. China

E-mail address: dingfan@math.pku.edu.cn

Mathematisches Institut, Universität Zu KÖln, Weyertal 86-90, 50931 Köln, GerMANY

E-mail address: geiges@math.uni-koeln.de 